\title{
The Effects of FSH and Glutathione Supplementation to the in Vitro Maturation Media on Mouse Oocyte Maturation
}

\author{
Roza Silvia ${ }^{1}$, Sally Catt ${ }^{2}$, Mulyoto Pangestu ${ }^{3}$ \\ \{roza.silvia_md@yahoo.com $\left.{ }^{1}\right\}$ \\ Department of Histology, Faculty of Medicine, Universitas Andalas ${ }^{1}$ \\ Monash Institute of Medical Research, Centre of Reproduction and Development, Monash \\ University, Australia ${ }^{2,3}$
}

\begin{abstract}
Either Follicle-stimulating hormone (FSH) or glutathione solely has been known to have benefits on oocyte maturation, yet study about combination effects are still limited. This study aimed to observe the effects of FSH and glutathione supplementation to the in vitro maturation (IVM) media on mouse oocyte maturation. Immature oocytes of F1 (C57BL/6xCBA) female mice were matured for 24 hours in Minimum Essential Medium (MEM) Alpha supplemented with $5 \mu \mathrm{g} / \mathrm{mL}$ FSH and $0.15 \mathrm{mM}$ glutathione, in 3 different combinations, i.e. the MEM alpha without any supplementations, supplementation of FSH solely, and combination of FSH with glutathione. A total of 209 immature oocytes were studied to examine the oocyte maturation. Treatment with FSH supplementation alone $(p=0.043)$ and in combination with glutathione $(p=0.016)$ showed significantly higher oocyte maturation. This study indicated that in vitro oocyte maturation can be augmented by supplementation of IVM medium with FSH, either solely or along with glutathione.
\end{abstract}

Keywords: FSH, Glutathione, In Vitro Maturation, Medium, Oocyte.

\section{Introduction}

Suboptimal composition in IVM, is believed as one of the factors related to the lower success rates of IVM. Based on several animal studies, various existing commercial IVM media show the different effects on oocytes maturation and the following preimplantation development [1]. In fact, it still becomes a critical challenge to reveal all the factors contributing to the competence of oocyte maturation and how the control mechanisms work [2]

One of the important components known for oocyte maturation is FSH. FSH may improve the oocyte maturation, fertilization and embryo development. It functions to enhance the maturation of oocytes in isolated cumulus-oocyte complexes (COCs), especially nuclear maturation [3]. The presence of FSH in IVM medium is considered favourable for oocyte quality [3][4][5]. Study by Lee et al (2007) indicated that cumulus cell expansion and nuclear in vitro maturation rate of canine oocyte can be promoted by FSH supplementation to IVM medium. Li et al (2013) signified that the varied concentration of FSH added to IVM medium resulted in different outcomes. According to them, addition 50 or 100 IU FSH to IVM medium exhibited significantly higher on in vitro oocyte maturation when compared with supplementation of 200 IU. Still, the most suitable concentration remains unclear [6]. 
The importance of reduced form of glutathione for in vitro oocyte maturation has been also reviewed in several studies. It is considered as a strong antioxidant. It may limit free radicals produced in IVM process and prevent the impacts of oxidative stress (OS) on the oocytes, such as prematuration arrest and chromosomal segregation [7]. Naturally, the presence of cumulus cells protects the oocytes from OS through gluthatione synthesis [8].Tatemoto et al (2000) suggested that during IVM, greater DNA damage due to reactive oxygen species (ROS) may occur on denuded-oocytes when compared with COC because of the absence of cumulus cells [9] . In addition, Curnow et al (2011) reported the improving of early and late macaque oocyte maturation after gluthatione addition to the IVM medium.[10]

Tabel 1. Compound of IVM medium [11]

\begin{tabular}{|c|c|c|c|c|c|c|}
\hline \multirow[t]{2}{*}{ Compound $(\mathrm{mmol} / \mathrm{l})$} & \multicolumn{6}{|l|}{ Medium } \\
\hline & TCM199 & Waymouth MB 752/1 & Ham's F-12 & MEM & DMEM & $\mathrm{HECM}$ \\
\hline $\mathrm{CaCl}_{2}$ & 1.802 & 0.82 & 0.23 & 1.36 & 1.36 & 1.9 \\
\hline $\mathrm{MgSO}_{4}$ & 0.788 & 3.96 & 0.58 & 0.79 & 0.79 & \\
\hline $\mathrm{KCl}$ & 5.367 & 2.01 & 3 & 5.37 & 5.37 & 3 \\
\hline $\mathrm{NaCl}$ & 116.359 & 102.67 & 130.05 & 116.36 & 109.51 & 113.8 \\
\hline $\mathrm{NaHCO}_{3}$ & & 26.66 & 14 & 26.19 & 44.04 & 25 \\
\hline $\mathrm{Na}_{2} \mathrm{HPO}_{4}$ & 1.017 & 2.5 & 1.18 & 1.17 & 1.04 & \\
\hline DL-alanine & 0.561 & & 0.1 & & & \\
\hline L-arginine & 0.332 & 0.36 & 1 & 0.6 & 0.4 & \\
\hline DL-aspartic acid & 0.451 & 0.45 & 0.1 & & & 0.01 \\
\hline Asparagine & & & & & & 0.01 \\
\hline L-cysteine & $6.98 \times 10^{-4}$ & 0.51 & 0.22 & & & 0.01 \\
\hline L-cystine & 0.083 & 0.06 & & 0.1 & 0.2 & \\
\hline DL-glutamic acid & 0.908 & 1.02 & 0.1 & & & 0.01 \\
\hline L-glutamine & 0.684 & 2.4 & 1 & 2 & 4 & 0.2 \\
\hline Glycine & 0.666 & 0.67 & 0.1 & & 0.4 & 0.01 \\
\hline L-histidine & 0.104 & 0.78 & 0.17 & 0.2 & 0.2 & 0.01 \\
\hline Hydroxy-L-proline & 0.0763 & & & & & \\
\hline DL-isoleucine & 0.305 & 0.19 & 0.03 & 0.4 & 0.8 & \\
\hline DL-leucine & 0.915 & 0.38 & 0.1 & 0.4 & 0.8 & \\
\hline L-lysine & 0.479 & 1.64 & 0.25 & 0.5 & 1 & 0.01 \\
\hline DL-methionine & 0.201 & 0.34 & 0.03 & 0.1 & 0.2 & \\
\hline DL-phenylalanine & 0.303 & 0.3 & 0.03 & 0.2 & 0.4 & \\
\hline L-proline & 0.348 & 0.43 & 0.3 & & & 0.01 \\
\hline DL-serine & 0.476 & & 0.1 & & 0.4 & 0.01 \\
\hline Taurine & & & & & & 0.5 \\
\hline DL-threonine & 0.504 & 0.63 & 0.1 & 0.4 & 0.8 & \\
\hline DL-tryptophan & 0.0979 & 0.20 & 0.01 & 0.05 & 0.08 & \\
\hline L-tyrosine & 0.256 & 0.26 & & 0.23 & 0.46 & \\
\hline DL-valine & 0.427 & 0.56 & 0.1 & 0.4 & 0.8 & \\
\hline Glucose & 5.55 & 27.75 & 10 & 5.55 & 24.97 & \\
\hline DL-lactate & & & & & & 4.5 \\
\hline Pyruvate & & & & & 1 & \\
\hline Glutathione & $1.62 \times 10^{-4}$ & 0.16 & & & & \\
\hline Hypoxanthine & 0.0022 & 0.18 & 0.04 & & & \\
\hline
\end{tabular}

TCM =tissue culture medium; MEM = Minimum Essential Medium; DMEM=Dulbecco's modification of Eagle's medium; mBM-3=Basic salt medium 3; HECM $=$ hamster embryo culture medium.

Since not all commercial IVM media provide those two components in their composition [11], it is still questionedhow the effects of that combination on oocyte maturation in vitro. As known advantages of FSH and gluthationefor oocyte maturation, we hypothesized that modification of IVM media by FSH and glutathione supplementation may increase the IVM success rate. This study was undertaken to observe the effects of FSH and glutathione supplementation to IVM medium on oocyte maturation and embryo development 


\section{Materials and methods}

\subsection{Mice and replicates}

F1 (C57BL/6xCBA) female mice were used in this project. Mice were housed in Animal House Monash Medical Center (MMC) and maintained in accordance with associated codes of practice. All animals were approved by the MMC Animal Ethics Committee 'A' under approval no. MMC 2011/84.There were six replicates overall for in vitro oocyte maturation observation. Each replicate involved two mice. Priming and oocyte collecting were done in the afternoon time, almost in the same time for each replicate.

\subsection{Media preparation}

IVM base medium used was Minimum Essential Medium (MEM) Alpha (Gibco, Invitrogen Corporation) supplemented with $25 \mathrm{mM}$ sodium bicarbonate, $2 \mathrm{mM}$ L-alanyl-L-glutamine (GlutaMAX ${ }^{\mathrm{TM}}$, Gibco, Invitrogen Corporation), $1 \%$ penicillin/streptomycin (Gibco, Invitrogen Corporation), and $0.4 \% \mathrm{w} / \mathrm{v}$ bovine serum albumin (BSA). $\mathrm{pH}$ was adjusted to 7.8-7.9 and osmolarity was within $280 \pm 5$. It was then supplemented with $5 \mu \mathrm{g} / \mathrm{ml} \mathrm{FSH}$ (Folltropin ${ }^{\circledR}$, Bioniche Animal Health Canada Inc.), and $0.15 \mathrm{mM}$ L-Glutathione reduced (Sigma Chemical Co.) according to 3 different treatments as shown in table 2. For handling medium, MEM (HEPES, Gibco, Invitrogen Corporation) was supplemented with $0.3 \%$ w/v BSA, 2 mM Lalanyl-L-glutamine and $1 \%$ penicillin/streptomycin. $\mathrm{pH}$ ranged between 7.2-7.4 and osmolarity was between 260-280. All IVM media and handling media were prepared fresh weekly. Fertilization medium used was Quinn's Advantage ${ }^{\circledR}$ Fertilization (HTF; Sage, In-vitro Fertilization, Inc., Trumbull) supplemented with 2mM L-alanyl-L-glutamine.

Table 2. IVM treatments used in this project

\begin{tabular}{lll}
\hline Treatment & Supplementation & Final concentration* \\
\hline I & - & - \\
II & FSH & $5 \mu \mathrm{g} / \mathrm{ml}$ \\
III & FSH & $5 \mu \mathrm{g} / \mathrm{ml}$ \\
& Glutathione & $0.15 \mathrm{mM}$ \\
\hline
\end{tabular}

* osmolarity was assured to be within the range

\subsection{Priming and embryo collection}

5 IU pregnant mare's serum gonadotropin (PMSG; Folligon, MSD Animal Health, Bendigo) was injected (intra-retroperitoneal) to female mice. 48 hours later, the mice were killed by cervical dislocation. The ovaries were removed and transferred to sterile tubes with warmed handling medium. To retrieve immature oocytes, large antral follicles were punctured with sterile needles no. 29 G. Immature oocytes collected were all in germinal vesicles (GV) stage and categorized as shown in Table 3. The sample of pictures can be seen in figure 1. Immediately, all immature oocytes collected were placed into fresh handling media, then 
washed once through IVM base medium and finally divided equally into three different IVM treatment groups.

Table 3. Immature oocytes' categories

\begin{tabular}{ll}
\hline Category & Explanation \\
\hline $\begin{array}{l}\text { nude cumulus-oocyte } \\
\text { complex (n-COC) }\end{array}$ & None or very few cumulus cells $(<10)$ surrounding immature oocyte \\
partial COC (p-COC) & $\begin{array}{l}\text { Several layers of cumulus cells partially surrounding immature } \\
\text { oocyte }\end{array}$ \\
full COC (f-COC) & \begin{tabular}{l} 
Several layers of cumulus cells completely surrounding oocyte \\
\hline
\end{tabular}
\end{tabular}

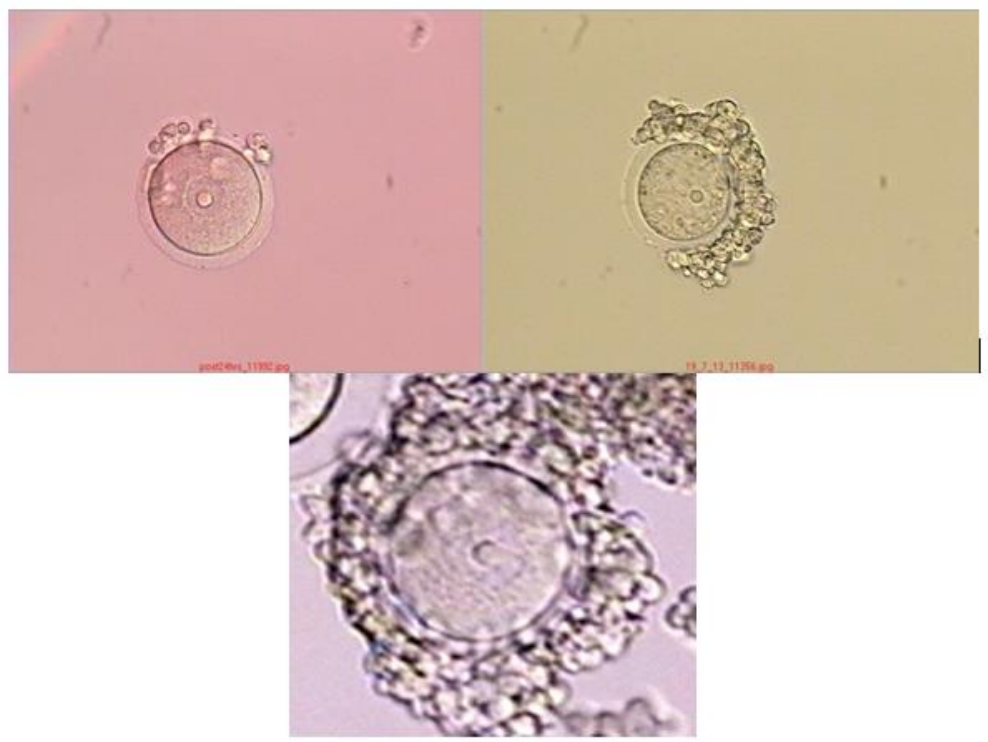

Fig. 1. Immature oocyte collected A. nude COC; B. partial COC; C. full COC

\subsection{Maturing oocytes in vitro}

Immature oocytes were put into 4-well Nunc dish with IVM media according to three different treatments (after overnight incubation with $37^{\circ} \mathrm{C}, 5 \% \mathrm{CO} 2$ ) for $24-26$ hours. Maturation was indicated by expansion of cumulus cells, or the presence of first polar body (PB I).

\subsection{Statistical analysis}

Statistical analysis was performed using Chi-square test to evaluate the effect of FSH and gluthatione supplementation in IVM medium to maturation rate. Statistically significant difference was defined as $\mathrm{p}$ value $<0.05$. 


\section{Result}

\subsection{In vitro oocyte maturation rate}

Overall, 209 immature oocytes were retrieved from six replicates. In general, $88(42.10 \%)$ were nude, $70(33.49 \%)$ were partial COC and 51 (24.41\%) were full COC. A distribution of oocyte type is given in table 4. Maturation of oocytes correalates significantly with FSH and gluthatione supplementation to the IVM medium ( $\mathrm{p}=0.043$ and $\mathrm{p}=0.016$ respectively for treatment 2 and 3 ).

Table 4. Distribution of immature oocyte number and in vitro maturation rate*

\begin{tabular}{lccccc}
\hline \multirow{2}{*}{ Treatment** } & \multicolumn{3}{c}{ Immature oocyte collection } & \multicolumn{2}{c}{$\begin{array}{c}\text { Mature oocyte } \\
\text { Number (rate) }\end{array}$} \\
\cline { 2 - 5 } I & n-COC & p-COC & f-COC & Total & Num \\
II & $30(42.86 \%)$ & $24(34.28 \%)$ & $16(22.86 \%)$ & 70 & $15(21.43 \%)$ \\
III & $29(41.42 \%)$ & $23(32.85 \%)$ & $18(25.73 \%)$ & 70 & $26(37.14 \%)$ \\
Total & $29(42.02 \%)$ & $23(33.33 \%)$ & $17(24.65 \%)$ & 69 & $29(42.03 \%)$ \\
\hline
\end{tabular}

* from six replicates** Treatment I $=$ MEM- $\alpha$ without any supplementation; Treatment II $=$ MEM- $\alpha$ with FSH supplementation; Treatment III = MEM- $\alpha$ with FSH and glutathione supplementation.

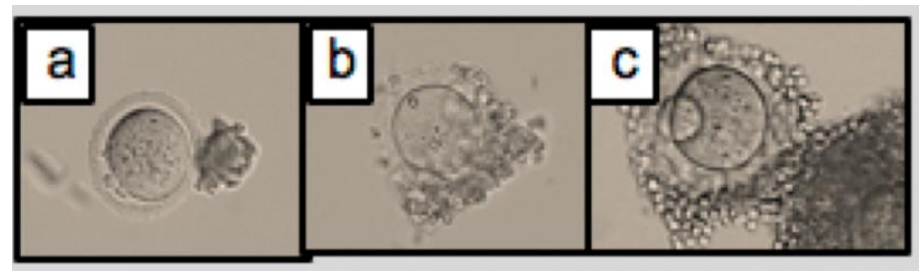

Fig. 2. Mature oocytes A. PB I extruded; B dan C. expansion of COC

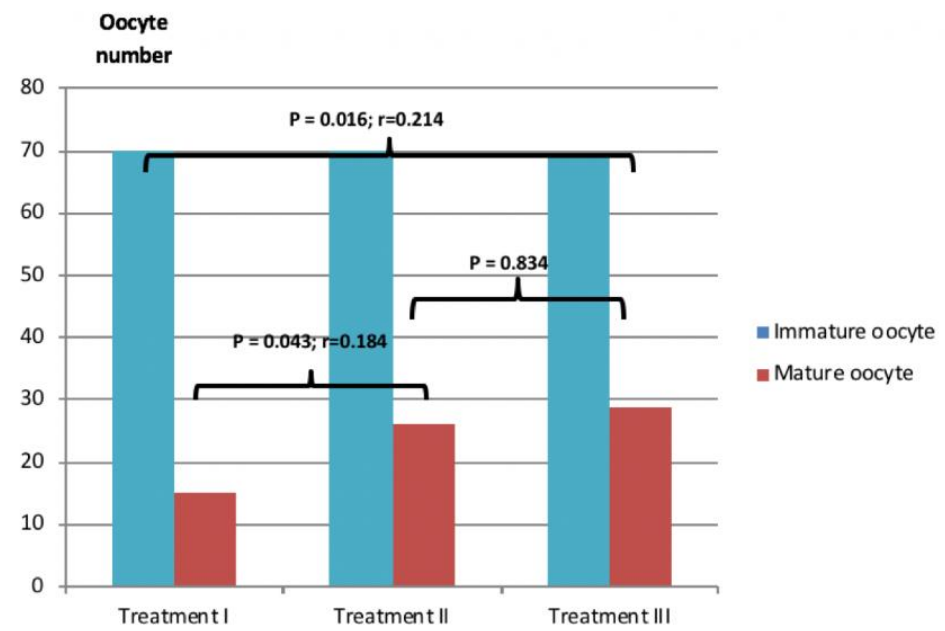

Fig. 3. Proportion of in vitro oocyte maturation between different treatments of IVM media 


\section{Discussion}

The results have indicated that single supplementation of FSH $(5 \mu \mathrm{g} / \mathrm{ml})$ to MEM alpha medium showed a significant difference of in vitro oocyte maturation rate achieved from the treatment without FSH supplementation $(P$ value $<0.05)$. This finding supports our hypothesis that FSH supplementation to IVM medium may enhance the in vitro oocyte maturation. The result is also in accordance with the previous study involving mouse oocytes done by Eppigg et al (2000). Besides, study on bovine oocytes by Ali and Sirard (2005) also highlighted the good effects of FSH addition to maturation culture media. Another study has proven that FSH receptor mRNA exists only in granulosa cells and cumulus cells, not in oocytes isolated from bovine antral follicles (van Tol et al, 1996). It improves GV breakdown in COC, but not in denuded oocytes. It is also considered to produce the substance that may accelerate oocyte meiotic resumption (Byskov et al, 1997). Hence, its supplementation to MEM alpha medium is beneficial. [12],[13],[14],[15]

This project also revealed that addition of $0.15 \mathrm{mM}$ glutathione to IVM medium, along with FSHshowed a significant difference of in vitro oocyte maturation rate achieved from the treatment without FSH supplementation $(P$ value $<0.05)$. Similar to this finding, IVM medium supplemented with $5 \mathrm{mM}$ of glutathione ethyl ester had significantly increased the IVM rates of Macaque oocytes [10]. Supplementation gluthationeto the IVM base medium showed its benefit to the oocyte maturation as it acts as the antioxidant agent that prevents the oocytes from OS.

It is known that oocyte maturation, either in vivo or in vitro, should gain both nuclear and cytoplasmic maturation. As cytoplasmic maturation is characterized by some ultra-structural and biochemical changes in oocyte cytoplasm that are necessary for fertilization and further embryo development, it will also influence the oocyte competence in successful fertilization and corresponding embryo development [12],[16]. Miron (2006) highlighted the less competence in cytoplasmic maturation of IVM oocyte compared to in vivo matured oocyte as the cause of low developmental rates of IVM oocytes after cleavage, while the nuclear maturation is alright. [17]

This study had several limitations. Regarding the methods, they were also restricted to only one determined concentration of any supplementations added with only simple combinations of treatments arranged. Indeed, the effect of those supplementations could not be analysed in more details. Moreover, whether different concentrations and combinations may affect the results could not be revealed. No detail evaluations on cytoplasmic maturation should also be improved in the future study for more details. Besides, no separated analysis run on three different groups of immature oocytes collected made it unable to evaluate the influence of cumulus cells to in vitro oocyte maturation in connection with the work mechanism of FSH and/or any other supplements.

\section{Conclusion}

In conclusion, this study demonstrated that in vitro oocyte maturation can be augmented by supplementing IVM medium with FSH, either solely or with glutathione. Finally, for the future study, improvement for limitations in this study would help to get a better and more reliable result. 


\section{References}

[1] Robert R, Franks S, Hardy K. Culture environment modulates maturation and metabolism of human oocytes. Human Reproduction. 2002; 17 (10): 2950-2956.

[2] Gilchrist RB, Thompson JG. Oocyte maturation: emerging concepts and technologies to improve developmental potential in vitro. Theriogenology 2007; 67: 6-15.

[3] Sha W, Xu B-Z, Li M, Liu D, Feng HL, Sun Q-Y. Effect of gonadotrophins on oocyte maturation in vitro: an animal model. Fertility and Sterility 2010; 93 (5): 1650-1661.

[4] Lee HS, Seo YI, Yin XJ, Cho SG, Lee SS, Kim NH, Cho SK, Kong IK.Effect of follicle stimulation hormone and luteinizing hormone on cumulus cell expansion and in vitro nuclear maturation of canine oocytes.ReprodDomest Anim. 2007; 42(6):561-5.

[5] Sirard MA, Desrosier S, Assidi M. In vivo and in vitro effect of FSH on oocyte maturation and developmental competence. Theriogenology, 2007; 68S: S71-S76.

[6] Li M, Zhao Y, Zhao CH, Yan J, Yan YL, Rong L, Liu P, Feng HL, Yu Y, Qiao L. High FSH decrease the development al potential of mouse oocytes and resulting fertilized embryos, but does not influence offspring physiology and behaviour in vitro or in vivo. Human Reproduction 2013.

[7] Combelles C. Could oxidative stress influence the in-vitro maturation of oocytes? Reproductive Biomedicine Onnline 2009; 18 (6): 864-880.

[8] Abeydeera LR, Wang W-H, Cantly TC, Prather RS, Day BN. Glutathione content and embryo development after in vitro fertilization of pig oocytes matured in the presence of a thiol compound and various concentrations of cysteine. Zygote 1999; 7: 203-210.

[9] Tatemoto H, Sakurai N, Muto N. Protection of porcine oocytes against apoptotic cell death caused by oxidative stress during in vitro maturation: role of cumulus cells. Biology of Reproduction 2000; 63: 805-810.

[10] Curnow EC, Ryan JP, Saunders DM, Hayes ES. Primate model of metaphase I oocyte in vitro maturation and the effects of a novel glutathione donor on maturation, fertilization, and blastocyst development. Fertility and Sterility 2011; 95 (4): 3: 1235-1240.

[11] Sutton-McDowall ML, Gilchrist RB, Thompson JG. Effects of in-vivo and in-vitro environments on the metabolism of the cumulus-oocyte complex and its influence on oocyte developmental capacity. Human Reproduction Update 2003; 9 (1): 35-48.

[12] Eppig JJ, Hosoe M, O’Brien MJ, Pendola FM, Requena A, Watanabe S. Condition that affect acquisition of developmental competence by mouse oocytes in vitro: FSH, insulin, glucose and ascorbic acid. Mol Cell Endocrinol 2000; 163: 109-116.

[13] Ali A, Sirard MA. Protein kinases influence bovine oocyte competence during short-term treatment with recombinant human follicle stimulating hormone. Reproduction 2005; 130: 303 310.

[14] Byskov AG, Yding Andersen C, Hossaini A, Guoliang X. Cumulus cells of oocyte-cumulus complexes secrete a meiosis-activating substance when stimulated with FSH. MolReprod Dev 1997; 46: 296-305.

[15] Van Tol HT, van Eijk MJ, Mummery CL, van den Hurk R, Bovers MM. Influence of FSH and hCG on the resumption of meiosis of bovine oocytes surrounded by cumulus cells connected to membrane granulosa. MolReprod Dev 1996; 45: 218-224.

[16] Sathananthan AH. Ultrastructure of the human egg. Human Cell. 1997: 10; 21-38.

[17] Miron P. In-vitro maturation of oocytes: biological aspects. Reproductive BioMedicine Online. 2006: 13 (3); 437-446. 